\title{
Cervezas elaboradas artesanalmente: análisis de la normativa técnico-sanitaria vigente
}

\author{
Handcrafted beer: analysis of applicable sanitary technical regulations
}

\author{
Bigeon G*, Benítez F, Pellicer K, Copes J \\ Cátedra de Tecnología y Sanidad de los Alimentos, Departamento de Epizootiología y Salud Pública. \\ Facultad de Ciencias Veterinarias, Universidad Nacional de La Plata. \\ *Correo electrónico del autor: gbigeon@fcv.unlp.edu.ar
}

\begin{abstract}
Resumen: La cerveza es tan antigua como nuestra civilización. Actualmente, ha surgido una gran variedad de cervezas en América del Sur, alguna con características verdaderamente atípicas o diferentes con respecto al concepto clásico, principalmente elaboradas por el sector "microcervecero" y/o "artesanal". Este trabajo consistió en la búsqueda, recopilación y análisis de la normativa técnicosanitaria vigente para este tipo de productos en la región. Argentina cuenta con una norma vertical dentro del Código Alimentario Argentino para cervezas «elaboradas artesanalmente», debiendo cumplir también con las normas horizontales para cerveza establecidas por el MERCOSUR. En la actualidad, este es el único país en el bloque regional que cuenta con legislación específica para estos productos. Brasil y Bolivia cuentan con anteproyectos de normas para definir el producto y los establecimientos, mientras que el resto de los estados parte no poseen normativa vertical. Colombia posee un registro de este tipo de productos incluyendo en el rótulo el término "artesanal". Chile, Ecuador y Perú no cuentan actualmente con ninguna reglamentación que haga referencia a las cervezas elaboradas de forma artesanal. Se concluye que sería conveniente armonizar criterios o establecer parámetros técnicos específicos para las cervezas elaboradas de forma artesanal en la región, indistintamente de cual sea su jurisdicción, sin que esto implique el incumplimiento de la norma general para cerveza. Esta propuesta tiene como objeto resguardar la Salud Pública, garantizar una leal competencia entre los elaboradores y proporcionar información adecuada a los consumidores mediante la correcta rotulación.
\end{abstract}

Palabras clave: cerveza, elaboración artesanal, normativa técnica, América del Sur

\begin{abstract}
Beer is as old as our civilization. Nowadays, a wide range of beers has emerged in South America, some of them with truly atypical or diverse characteristics compared to the classic concept, manufactured mainly by the "micro brewery" and/or "craft brewery" sector. This work consisted in the search, compilation and analysis of the technical-sanitary norms applicable to this type of products in the region. Argentina has vertical regulations within the Argentine Food Code for "craft beers", which still requires compliance with horizontal regulations for beers established by MERCOSUR. Currently, this is the only country within the regional bloc that has specific legislation for these products. Brazil and Bolivia have regulatory projects to define the product and facilities, while the rest of the member states do not have vertical regulations. Although Colombia has a registry for this type of products, it includes the term "crafts" on its label. Chile, Ecuador and Peru do not currently have any regulations regarding craft beers. In conclusion, it would be wise to harmonize the criteria or establish specific technical standards for craft beers in the region, regardless of their scope, without this implying the breach of the general rule for beers. The aim of this proposal consist to ensure the protection of public health, fair competition among brewers and provide adequate information to consumers at the time of purchase through accurate labeling.
\end{abstract}

Key words: beer, craft beers, technical regulations, South America 


\section{Introducción}

La cerveza, bebida alcohólica elaborada a partir de agua, cereal, lúpulo y levadura, es tan antigua como nuestra civilización. Se cree que su origen está unido a los primeros asentamientos humanos junto al desarrollo de la agricultura y al alejamiento del modo de vida nómade (FIFCO). Se considera que los sumerios fueron los primeros en fermentar granos como la cebada. Fue en el desierto de Sumeria (Mesopotamia asiática), hace unos 6.000 años, donde se halló el registro sobre unas tablas de arcilla con las primeras menciones de la cerveza, llamada sikaru (Alcazar, 2001).

Esta bebida ha ido modificándose a lo largo del tiempo, beneficiándose con los progresos científicos y técnicos, logrando, de esta forma, un avance y mejora tecnológica, como por ejemplo, la automatización de sus procesos (Carrillo, 2009; FIAB, 2009). Algunos de ellos son: la reducción de la oxidación antes y durante la maceración, la disminución en el contenido de sustancias volátiles que afectan la calidad del producto, la restricción del consumo de energía en la elaboración del mosto (para mermar el costo de producción y mejorar la calidad del producto) y la implementación de medidas para contribuir a la gestión de los residuos cerveceros de elaboración (Carrillo, 2009).

Se estima que la difusión del consumo de cerveza en Latinoamérica está relacionada con la migración de habitantes extranjeros y su influencia (Cerpa \& Melo, 2011). En 2015, ocho países de la región concentraban la mayor parte de la producción de cerveza (291 millones de hectolitros), liderados por Brasil, México seguidos por otros tres países con una cantidad similar: Venezuela, Colombia y Argentina (Petevel, 2016).

El consumo de cerveza en Argentina es de 44,7L per cápita por año. Esta cifra es la más alta entre todas las bebidas alcohólicas que se consumen (Ablin, 2014), ocupando el puesto 72 del ranking mundial. El ranking está encabezado por la República Checa (147L), Alemania (111L) y Austria (109L). Con respecto a las ventas, Argentina comercializa 17 millones de hectolitros de cerveza por año, lo que permite considerarlo como uno de los países con mayor potencial de crecimiento (La Capital, 2016).

En la actualidad, han surgido una gran variedad de cervezas en la región, alguna de ellas con características verdaderamente atípicas o diferentes respecto del concepto clásico, principalmente elaboradas por el sector "microcervecero" y/o "artesanal” (Bascur Palacios, 2013). El volumen de producción, la tecnología implementada, las materias primas utilizadas, la receta del maestro cervecero $y$, principalmente, las diferencias en el proceso de elaboración, favorecen el surgimiento de una gran multiplicidad de estilos (Martínez Muñoz, 2015).
En cuanto a los productos, se puede resaltar que son relativamente nuevos, variados, y sus características difieren en volumen, materias primas, composición, color, elaboración o producción, forma de gasificación, entre otras. Esto hace que sea difícil unificar criterios y establecer pautas de índole técnico.

Con respecto a los establecimientos elaboradores de "cervezas artesanales", encuadrarlos y definirlos dentro de un reglamento es un trabajo arduo y engorroso, en el que idealmente quedarían plasmadas las cuestiones operativas, técnicas y edilicias que estos deben cumplir. Los requerimientos deberían ser contemplados siempre y cuando cumplan con los requisitos de seguridad e higiene mínimos establecidos para garantizar la inocuidad del producto. Por lo tanto, establecer las características de las "cervecerías artesanales" y consignar las diferencias con las industriales no es tarea sencilla, como por ejemplo, en la definición de la cantidad de litros producidos por el establecimiento, es decir, las escalas de producción.

El consumo de esta bebida se ha ido incrementando debido a cambios sociales y de estilo de vida. El consumidor busca calidad y diferentes variedades de cervezas, lo que ha provocado un aumento de pubs y bares que promueven su consumo y en lo que lo social juega un rol fundamental (Martínez Muñoz, 2015).

En nuestro país se observa que las "cervecerías artesanales" han proliferado (20\% anual) (Ablin, 2014) $\mathrm{y}$, junto con la venta de hamburguesas, son los negocios de moda que registran un crecimiento exponencial de ventas (La Nación, 2017), con un crecimiento en variedades y productos que hasta hace poco no existían ni se elaboraban (Ablin, 2014).

En Argentina, las cervezas cuentan con un marco legal y técnico a cumplir dentro del Código Alimentario Argentino (CAA) en el que se ha incorporado en su articulado el reglamento técnico MERCOSUR para este tipo de productos. Recientemente, se ha establecido legislación relativa a las cervezas "elaboradas artesanalmente", la que nace de la necesidad de contar con las herramientas legales para legitimarlas.

El objetivo del presente trabajo consistió en la búsqueda y recopilación de la normativa técnicosanitaria en América del Sur, vigente al 1 de junio de 2017 , en relación con las cervezas elaboradas bajo metodología "artesanal" y su comparación con la situación técnico-legal de la cerveza.

\section{Normas analizadas}

Se llevó a cabo un análisis de la normativa sanitaria para las cervezas, con hincapié en las elaboradas artesanalmente. Los elementos para la realización del análisis comprendieron las normas encontradas y 
vigentes a la fecha de corte: 1 de junio de 2017; también se consignaron dos anteproyectos de ley (Brasil y Bolivia), no vigentes en la actualidad.

Resolución GMC №14/01, Reglamento técnico del MERCOSUR para productos de cervecerías.

Código Alimentario Argentino (CAA), Capítulo XIII, artículos 1080, 1081,1082, y 1083.

Resolución conjunta SPReY y SV N5-E/ 2017 que dictamina la incorporación al CAA del artículo $\mathrm{N}^{\circ}$ 1082 bis.

Decreto 6871/09, Brasil Diário Oficial da União - Seção 1 - 5/6/2009, Página 20 (Publicação Original). Disponible en: http://www2.camara.leg.br/legin/fed/decret/2009/decreto-6871-4-junho-2009-588673-normape-html.

Proyecto de Ley $\mathrm{N}^{\circ} 5191 / 13$, regulación de cervezas artesanales. Brasil. Disponible en: http://www. cerevisiaie.com.br/guía-regulamentacao-cervejaartesanal.

Norma Boliviana NB 382/2001, productos de cervecería, IBNORCA. Bolivia. Disponible en: http:// www.ibnorca.org/images/archivos/IBNORCACATALOGODENORMAS2016Noviembre.pdf.

Anteproyecto de norma boliviana, relacionada a las cervezas artesanales APNB 323001(16) Disponible en: http://www.ibnorca.org/images/archivos/consultasept15/Septiembre2016/APNB323001. pdf.

Ley $N^{\circ} 18455$, con su Decreto Supremo reglamentario $\mathrm{N}^{\circ} 78$ del 1986, última modificación: Diario Oficial $n^{\circ} 39.290$ del 17 de febrero de 2009. Chile.

Decreto $N^{\circ} 1686$ del año 2012, Colombia. Diario Oficial No. 48.517 del 9 de agosto de 2012.

Norma Técnica Colombiana NTC 3854. Bebidas alcohólica, Cerveza. 1996-03-02.

Norma Técnica Obligatoria $\mathrm{N}^{\circ} 2262$, Ecuador. El Consejo Directivo del INEN aprobó este proyecto de norma en sesión el 2 de agosto de 2002.

Norma Técnica Peruana 213.014:2016, Perú. El Peruano. Publicación Oficial - Diario Oficial, 14 de abril de 2016.

Decreto Supremo 20-90-ICTIIND, Perú. El Peruano, 21 de junio de 1990.

\section{Situación en diversos países de la región}

Argentina, Brasil, Paraguay, Uruguay y Venezuela integran el bloque regional llamado MERCOSUR. Este cuenta con la resolución GMC $N^{\circ} 14 / 01$, reglamento técnico MERCOSUR de productos de cervecería. En este documento, se fija la identidad y calidad de los productos de cervecería para el consumo humano, sin mencionar a las cervezas elaboradas en forma artesanal.
En Argentina, recientemente, se sancionó una norma para las "cervezas elaboradas artesanalmente", con fecha de publicación 3 de febrero de 2017 en el Boletín Oficial y entrada en vigencia al día siguiente. La Secretaría de Políticas, Regulación e Institutos y la Secretaría de Agregado de Valor, en resolución conjunta $N^{\circ}$ 5-E/ 2017, resolvieron en el: "ARTíCULO $1^{\circ}$ - Incorpórese al Código Alimentario Argentino el artículo 1082 bis, el que quedará redactado de la siguiente manera: "Podrá incluirse la leyenda 'Elaboración Artesanal' en el rótulo de aquella cerveza que cumpla con las siguientes exigencias: a) Que no utilice en su producción aditivos alimentarios; b) Que se encuentre adicionada únicamente con ingredientes naturales; c) Que la elaboración sea de manera manual o semiautomática; y d) Que en el caso que se le agregue jugos o extractos de frutas, éstos sean previamente pasteurizados". Además, se destaca que "a la cerveza que se comercialice con la leyenda "Elaboración Artesanal" no se le aplicará el parámetro de turbidez establecido en el artículo 1082 inciso b). Se permitirá el uso del gas autorizado en el artículo 1067". Cabe remarcar que esta reglamentación establece cuáles son las condiciones necesarias que debe cumplir el producto para poder llevar impreso en su etiqueta la frase "elaboración artesanal", generando la posibilidad de que las empresas rotulen legítimamente sus bebidas al incorporar la leyenda en su rótulo.

En Brasil, en 2013 se propuso un anteproyecto de ley $N^{\circ}$ 5191/13 (Cerevisiae, 2016), con el exclusivo objetivo de regular la producción de cerveza artesanal. La idea del proyecto es definir a la "cerveza artesanal" e instaurar las características de los establecimientos que producirían este tipo de producto, para ofrecer simplificaciones en el proceso de registro e inspección. Sin embargo, este proyecto se encuentra actualmente postergado, debatiéndose en las diversas comisiones, antes de ser sometida a votación (Rodrigues et al., 2014).

La norma 382 del año 2001 de Bolivia hace referencia a los productos de cervecería: definiciones, clasificación y requisitos. En relación a las "cervezas artesanales", este país cuenta con un anteproyecto de Norma IBNORCA, APNB 323001. La propuesta de este documento es establecer las definiciones, clasificación y los requisitos que deben cumplir las cervezas llamadas artesanales para su elaboración y/o comercialización en el territorio nacional boliviano. En la actualidad, este proyecto está a la espera de ser aprobado.

Chile cuenta con la Ley $N^{\circ} 18455$, que fija las normas sobre producción, elaboración y comercialización de alcoholes etílicos, bebidas alcohólicas y vinagres. Esta fue reglamentada por el Decreto Supremo $N^{\circ} 78$ de 1986, estableciendo las normas técnicas 
dispuestas para la elaboración de cerveza industrial y otras bebidas alcohólicas y vinagres. A partir del 2013, por la creciente actividad en el país para cervezas de elaboración artesanal, el Servicio Agrícola y Ganadero (SAG) tiene dentro de sus funciones fiscalizar la Ley de Alcoholes 18.455 en cuanto a su producción y comercialización y, en el marco de ello, el servicio entrega una acreditación para que los establecimientos elaboradores puedan funcionar. Por lo tanto, estos deben inscribir el producto y realizar análisis al mismo para comprobar graduación alcohólica y los ingredientes que se están utilizando (Martínez, 2013).

Colombia cuenta con el decreto $\mathrm{N} * 1686$ del año 2012 (Valencia et al., 2015) en el que se hallan los requisitos sanitarios que se deben cumplir para la fabricación, elaboración, hidratación, envase, almacenamiento, distribución, transporte, comercialización, expendio, exportación e importación de bebidas alcohólicas destinadas para consumo humano. Por otro lado, el decreto $N^{\circ} 3192$ de 1983, reglamenta parcialmente el Título $\vee$ de la Ley 9 de 1979, en lo que refiere a fábricas de alcohol y bebidas alcohólicas, elaboración, hidratación, envase, distribución, exportación, importación y venta de estos productos y establece mecanismos de control en el territorio nacional. También posee una Norma Técnica, NTC 3854, Bebidas Alcohólicas, cerveza. Hay antecedentes de que el Instituto Nacional de Vigilancia de Medicamentos y Alimentos - INVIMA, ha concedido registros sanitarios a "cerveza artesanal" rubia tipo belgian blond, quedando establecido el término cerveza artesanal en el rótulo. En la resolución $N^{\circ} 2014005947$ del 6 de marzo de 2014, por la cual se concede el Registro Sanitario, se consigna que el producto debe cumplir con el decreto $\mathrm{N}^{\circ} 1686$ del año 2012. Si bien en la etiqueta es posible expresar la denominación "cerveza artesanal", en dicho decreto no existen referencias ni definición del término "artesanal".

Ecuador posee la Norma Técnica (INEN) $\mathrm{N}^{\circ}$ 2262, "Bebidas Alcohólicas. Cerveza. Requisitos". En ella se establecen los requisitos que debe cumplir la cerveza para ser apta para el consumo humano. En el texto se definen los criterios físicos, químicos y microbiológicos. Los elaboradores de este tipo de bebidas artesanales se enfrentan, en el momento de la inscripción del producto, con una dificultad técnica y sanitaria específica: según la normativa, la cerveza debe ser pasteurizada, y la "cerveza artesanal" en Ecuador no lo es, sino que atraviesa otro proceso de procesamiento (e. comexplus, 2014). Por lo expuesto, no se garantiza el resguardo de la salud pública. Este es uno de los puntos importantes de conflicto a la hora de establecer la normativa técnica para las cervezas elaboradas artesanalmente, pues los muchos y variados procesos tecnológicos hacen a las características de las distintas cervezas. Debido a esto, las autoridades del INEN reconocen la necesidad de una ampliación de la normativa $\mathrm{N}^{\circ} 2262$ o inclusive una norma propia para las cervezas artesanales.

Perú posee varias normas técnicas (NTP) referidas a la cerveza. Cabe destacar que estas son voluntarias y están aprobadas a través de la Dirección de Normalización del Instituto Nacional de Calidad (INACAL). Mencionaremos la NTP 213.014:2016 Cerveza. Requisitos. $3^{\text {ra }}$ Edición que reemplaza a la NTP 213.014:2014. Este país cuenta con otras normas NTP, por ejemplo, la que tiene la finalidad de determinar la densidad relativa en cervezas. Por otro lado, el Decreto Supremo 20-90-ICTIIND establece la información que deberá llevar inscripta en el empaque, envase o etiqueta toda bebida alcohólica que sea puesta en venta. No se ha encontrado ninguna norma que haga referencia a las cervezas elaboradas artesanalmente en este país.

Sobre la bibliografía consultada, se remarca la reciente y escasa normativa para las cervezas "elaboradas artesanalmente" en la región; de hecho, cabe destacar la ausencia de legislación referida o definición establecida en el CODEX Alimentarius sobre este tipo de bebidas elaboradas en forma artesanal. No ocurre lo mismo con los productos de la cervecería en general o industriales, siendo estas normas utilizadas como marco legal y técnico en la actualidad. Esta situación trae como consecuencia la limitación a los productores para ampliar su rango de comercialización y resulta un obstáculo a la hora de solicitar permisos o habilitaciones y acceder a créditos.

Desde el punto de vista sanitario, es de suma importancia contar con requisitos bien definidos según el tipo de producción, en este caso "artesanal", así como los procedimientos de acuerdo con la normativa aplicable en materia de otorgamiento de permisos sanitarios de funcionamiento, autorizaciones sanitarias y de certificados de habilitación de establecimientos elaboradores.

\section{Discusión y conclusiones}

Dado el aumento en la producción y el consumo de cervezas artesanales basado en el cambio de hábitos sociales, Argentina cuenta con una norma vertical dentro de su CAA para cervezas "elaboradas artesanalmente" y no debe incumplir con las normas horizontales para cerveza establecidas por MERCOSUR e incorporadas en su código.

En el resto de los países la situación difiere, ya que predomina la escasa e incipiente normativa para cervezas elaboradas artesanalmente, a diferencia de la legislación para cervezas.

De lo expuesto, se concluye que sería prudente unificar criterios implementando una norma específica 
para cervezas elaboradas de forma artesanal en la región, sin dejar de cumplir con la norma general para cerveza. Esto garantizaría el resguardo de la Salud Pública y una leal competencia entre los elaboradores y, además, proporcionaría una adecuada información al consumidor en el momento de la compra mediante una correcta rotulación.

\section{Bibliografía}

Ablin A. 2014. Área de Sectores Alimentarios. Dirección de Agroalimentos. Secretaría de Agricultura, Ganadería y Pesca Informe Sectorial $N^{\circ} 3$. El Mercado de la Cerveza. Alimentos Argentinos. [ONLINE]. Disponible en: http://www. alimentosargentinos.gob.ar/contenido/sectores/bebidas/lnformes/Cerveza_03_2014_04Abr.pdf [Consultado 06/2017].

Alcazar RuedaA. 2001. Aplicaciones del análisis multivariante a la diferenciación de tipos de cerveza. Universidad de Sevilla. Facultad de Química. Departamento de Química Analítica. España. Pag 2. [ONLINE] Disponible en: http://fondosdigitales.us.es/media/thesis/1596/I_T-823introduccion.pdf [Consultado 08/2017].

APNB 323001. Anteproyecto de norma boliviana, relacionada a las cervezas artesanales.. [ONLINE]. Disponible en: http://www.ibnorca.org/images/archivos/consultasept15/ Septiembre2016/APNB323001.pdf [Consultado 05/2017].

Bascur Palacios GG. 2013. Plan de negocio de una cervecería artesanal en la región metropolitana. Memoria para optar al título de ingeniero civil industrial en Internet. Universidad de Chile, Facultad de Ciencias Físicas y Matemáticas. Departamento de Ingeniería Industrial. [ONLINE]. Disponible en: http://repositorio.uchile.cl/bitstream/handle/2250/113903/ cfbascur_gp.pdf?Sequence=1 [Consultado 06/2017].

Carrillo RU. 2009. Avances en la tecnología de elaboración de cervezas. Instituto de Investigaciones para la Industria Alimenticia. Congreso Nacional de biotecnología y bioingeniería. VII simposio Internacional de producción de alcoholes y levaduras. Pág. 1. Acapulco, México. [ONLINE]. Disponible en: http://www.smbb.com.mx/congresos $\% 20$ smbb/acapulco09/TRABAJOS/simposios/simposio levaduras_bebidas/RAUL_CARRILLO.pdf [Consultado 06/ 2017].

Cerevisiae. 2016. Guía de reglamentación de la cerveza artesanal. Brasil. [ONLINE]. Disponible en: cerevisiaie. com.br/guía-regulamentacao-cerveja-artesanal [Consultado 05/2017].

Cerpa J, Melo O. 2011. Determinantes del precio en cervezas: aplicación del modelo de precios hedónicos. Economía Agraria. Volumen 15-2011. [ONLINE]. Disponible en: http:// www.aeachile.cl/docs/r15/6744\%20-\%204.pdf [Consultado 05/2017].

FIFCO. Cervezas y mucho más. Cervecería Costa Rica. [ONLINE]. Disponible en: http://www.florida.co.cr/files/ documents/177_es p_cervezaymuchoms.pdf [Consultado 06/2017].

Código alimentario argentino (Ley $\mathrm{N}^{\circ}$ 18284/69), artículos 1080, 1081, 1082, y 1083. Capítulo XIII. Bebidas fermentadas. CAA. [ONLINE]. Disponible en: http://www.anmat. gov.ar/alimentos/codigoa/Capitulo_XIII.pdf [Consultado 06/2017].

Decreto $N^{\circ} 6871 / 09$, Brasil. Diário Oficial da União - Seção
1 - 5/6/2009, Página 20 (Publicação Original). [ONLINE]. Disponible en: http://www2.camara.leg.br/legin/fed/decret/2009/decreto-6871-4-junho-2009-588673-norma-pe. html [Consultado 06/2017].

Decreto $N^{\circ} 1686$ del año 2012, Colombia. Diario Oficial No. 48.517 de 9 de agosto de 2012. [ONLINE]. Disponible en: https://redjurista.fcm.org.co/fcm/docs/decreto_1686_2012. htm [Consultado 05/2017].

Decreto Supremo 20-90-ICTIIND, Perú. El Peruano, 21 de junio de 1990.

e. comexplus online. 2014. Normativa amarga la cerveza "artesanal". Publicación 1/10/14. [ONLINE]. Disponible en: http://e-comex-plus.com/noticias/normativas- 'amargan'-lacerveza-artesanal [Consultado 06/2017].

FIAB, Fondo social europeo, INEM y Fundación tripartita. 2009. Documento de síntesis. Estudio sectorial de industrias de elaboración de bebidas. Página 22. [ONLINE]. Disponible en: http://www.fiab.es/archivos/documentoAutor/documentoautor_20090313104129.pdf [Consultado 05/2017].

La Capital. 2016. Un estudio revela cuántos litros de cerveza consumen los argentinos en promedio durante un año. Información general. [ONLINE]. Disponible en: http:// www.lacapital.com.ar/informacion-gral/un-estudio-revelacuantos-litros-cerveza-consumen-los-argentinos-promedioun-ano-n1196368.html [Consultado 05/2017].

La Nación. 2017. El lado oscuro de las cervecerías artesanales. Diario online. Sección Economía. [ONLINE]. Disponible en: http://repositori.udl.cat/bitstream/handle/10459.1/48689/ amartinezm. pdf?Sequence=1\&isallowed=y [Consultado 06/2017].

Ley $N^{\circ} 18455$. Fija normas sobre producción, elaboración y comercialización de alcoholes etílicos, bebidas alcohólicas y vinagres. Última modificación: Diario oficial n³9.290 de 17 de febrero de 2009. Chile. [ONLINE]. Disponible en: http://www.gie.uchile.cl/pdf/GIE_legislacion/Ley_18455.pdf [Consultado 04/2017].

Martínez C. 2013. SAG llama a regularizar producciones de cerveza artesanal en La Araucanía. Biobiochile. cl. [ONLINE]. Disponible en: http://www. biobiochile.cl/noticias/2013/06/16/ sag-llama-a-regularizar-producciones-de-cerveza-artesanalen-la-araucania.shtml [Consultado 06/2017].

Martínez MuñozA. 2015. Análisis comparativo de compuestos bioactivos en cerveza artesanal y cerveza industrial. Universidad de lleida, Facultad de Medicina grado en nutrición humana y dietética, España. Pág. 12-13. [ONLINE]. Disponible en: http://repositori.udl.cat/bitstream/handle/10459.1/48689/ amartinezm. pdf? sequence=1\&isallowed=y [Consultado 05/2017].

NB 382/2001. Norma Boliviana, productos de cervecería, IBNORCA. Bolivia. 2001. [ONLINE]. Disponible en: http:// www.ibnorca.org/images/archivos/IBNORCACATALOGODENORMAS2016Noviembre.pdf [Consultada 05/2017].

Norma Técnica Obligatoria $N^{\circ} 2262$, Bebidas alcohólicas. Cerveza. Requisitos. Ecuador. Aprobada en sesión de 2002-02-08. [ONLINE]. Disponible en: https://odaninkasiquito.files.wordpress.com/2015/08/inen-2-262-cerveza.pdf [Consultado 06/2017].

Norma Técnica Peruana 213.014:2016. Bebidas alcohólicas: cerveza. El Peruano. Publicación Oficial - Diario Oficial, 14 de abril de 2016. Perú. [ONLINE].. Disponible en: http://busquedas.elperuano.com. pe/normaslegales/aprueban-normas- 
tecnicas-peruanas-sobre-bebidas-alcoholicas-resolucionn-078-2012cnb-indecopi-838103-2/ [Consultado 06/2017].

NTC 3854. 1996. Norma Técnica Colombiana, Bebidas Alcohólicas, Cerveza. [ONLINE]. Disponible en: https:// tienda.icontec.org/wpcontent/uploads/pdfs/NTC3854.pdf [Consultado 05/2017].

Petevel P. 2016. El top ocho de los mayores productores de cerveza en Latinoamérica. Mercado técnica publicidad medios. [ONLINE]. Disponible en: https://www.merca20.com/ top-8-los-productores-cerveza-latinoamerica/ [Consultado 05/2017]

Resolución conjunta N5-E/ 2017 del SPRel y SAV. Argentina. [ONLINE]. Disponible en: http://www.anmat.gov.ar/ alimentos/codigoa/Capitulo_XIII.pdf [Consultado 06/2017].

Resolución N 14/01del GMC, Reglamento técnico del MERCOSUR para productos de cervecerías. 2001. [ONLINE]. Disponible en: http://www.puntofocal.gov.ar/doc/r_gmc_1401.pdf [Consultado 06/2017]

Resolución No. 2014005947. INVIMA. Por la cual se concede un Registro Sanitario. 6 de marzo de 2014. Colombia. [ONLINE]. Disponible en: http://web.sivicos.gov.co/registros/ pdf/1168123_2014005947.pdf [Consultado 06/2017].

Rodrigues CCP, Moretti BDA, Saron A. 2014.Evaluation of biogas coming from the anaerobic treatment of the wastewater of craft brewery production of Pilsen and Weiss beer. XXXIV Congreso Interamericano de Ingeniería Sanitaria y Ambiental. Monterrey, México. [ONLINE]. Disponible en: https://www.researchgate.net/publication/295400618_Evaluation_of_biogas_coming_from_the_anaerobic_treatment of_the_wastewater_of_craft_brewery_production_of_pilsen_and_weiss_beer [Consultado 06/2017].

Valencia JS, Castro AP, Ramírez MF. 2015. Proyecto de viabilidad y factibilidad para la creación de una empresa productora y comercializadora de cerveza artesanal. Universidad Tecnológica de Pereira. Facultad de Tecnología industrial. Colombia. Pág. 26. [ONLINE]. Disponible en: http://repositorio.utp.edu.co/dspace/bitstream/ handle/11059/5885/65811S479. pdf?sequence=1 [Consultado 05/2017]. 